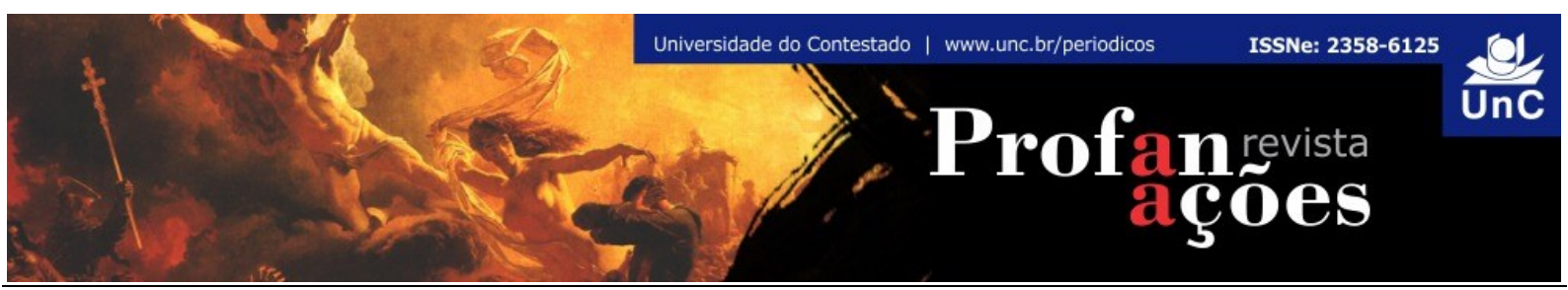

\title{
EL ESTADO DE EXCEPCIÓN Y SUS ARTICULACIONES CON UNA GENEALOGÍA DE LOS DESASTRES ECOSISTÉMICOS
}

RESUMEN: El presente artículo analiza la relevancia de la figura jurídica del estado de excepción en la obra filosófica de Giorgio Agamben. La primera parte examina las diferencias entre los institutos jurídicos del estado de excepción y la dictadura tomando en cuenta sus antecedentes en el derecho romano (homo sacer, iustitium, senatus consultum ultimum). En la segunda parte, se lleva adelante una arqueología del estado de excepción en sus relaciones con la oikonomia teológica medieval y el problema de los desastres ecosistémicos como parte de la gobierno moderno de las poblaciones. Finalmente, se considera la importancia de la cuestión de la extinción humana como horizonte político último del estado de excepción devenido regla en las sociedades globales contemporáneas.

Palabras-clave: Estado de excepción. Oikonomía. Desastres naturales. Extinción. Teología política.

\section{THE STATE OF EXCEPTION AND ITS ARTICULATIONS WITH A GENEALOGY OF ECOSYSTEMIC DESASTERS.}

\begin{abstract}
The following article analyses the relevance of the juridical figure of the state of exception in Giorgio Agamben's philosophical works. The first part examines the differences between the juridical institutes of the state of exception and dictatorship taking into account their background in ancient Roman Law (homo sacer, iustitium, senatus consultum ultimum). In the second part, an archaeology of the state of exception is carried out regarding its relationships with medieval theological oikonomía and the problem of the ecosystemic disasters as being both part of the modern government of populations. Finally, the importance of the question of human extinction is considered as the last political horizon concerning the state of exception transformed into a rule in the contemporary global societies.
\end{abstract}

Keywords: State of exception. Oikonomía. Natural disasters. Extinction. Political theology.

\footnotetext{
${ }^{1}$ Consejo Nacional de Investigaciones Científicas y Técnicas (CONICET); Universidad de Buenos Aires. Argentina. E-mail: fabianluduena@hotmail.com
} 


\section{EL ESTADO DE EXCEPCIÓN Y SUS ARTICULACIONES CON UNA GENEALOGÍA DE LOS DESASTRES ECOSISTÉMICOS}

Con un diagnóstico agudo que sigue la estela de un análisis puntilloso de la obra de Carl Schmitt, el filósofo Giorgio Agamben ha mostrado la estrecha vinculación existente entre el derecho público marcado por el principio según el cual "es soberano quien decide sobre el estado de excepción (Ausnahmezustand)" (SCHMITT, 2015, p. 11) y la progresiva horadación de los andamiajes jurídicos internacionales que han tendido, durante todo el siglo pasado y el presente, ha transformar el estado de excepción en una regla. Como ha sido demostrado, cuando Schmitt enunció su teoría sobre el estado de excepción, no sólo pensaba en la situación constitucional de Weimar o las dinámicas revolucionarias de la Europa posterior a 1848 sino, fundamentalmente, en una tesis política sobre el derecho público y la secularización (WALKER, 2005, p. 37).

De igual modo, Agamben ha querido señalar cómo el estado de excepción ha devenido, entonces, un paradigma de gobierno de la política contemporánea donde, al suspenderse la distinción entre los poderes legislativo, ejecutivo y judicial y, por consiguiente, al habilitarse la puesta entre paréntesis de la validez del derecho mismo, se ha abierto la puerta para una "guerra civil mundial" que gestiona la "(in)seguridad mundial" como la herencia actual de la gubernamentalidad (BIGO, 2005, p. 71-86).

La tesis de Agamben se basa en un exhaustivo análisis de la teoría de la excepción en el derecho público y en una arqueología filosófica de la noción en cuestión (AGAMBEN, 2004). Como ha sido reconocido, la teoría jurídica de la excepción comporta, en Agamben, una correlativa ontología política que no podemos analizar aquí pero que ha merecido sesudas pesquisas por parte de los especialistas (GULLì, 2007, p. 219-241). Sin duda, la preocupación por el problema de la "división de poderes" (HUMPHREYS, 2006, p. 684) suscitada entre los especialistas a partir de las proposiciones agambenianas se sustenta en la cercanía entre el estado de excepción como praxis de gobierno y la dictadura comisarial o soberana (SCHMITT, 1994).

En efecto, algunos teóricos habían intentado asimilar el estado de excepción a una especie de "dictadura perpetua" (REINACH, 1885, p. 41) mientras que otros 
buscaron abonar la idea de que, precisamente, la "dictadura constitucional" encontraba su punto de apoyo en "las ineficiencias inherentes a la doctrina de la separación de poderes" (ROSSITER, 1948, p. 288-290). No obstante, finalmente, los teóricos más sutiles mantienen la distinción entre el estado de excepción moderno y la institución de la dictadura, de origen indiscutiblemente ligado a la jurisprudencia romana antigua (FRIEDRICH, 1958, p. 413-414)2.

En efecto, mucho se ha escrito acerca de los límites de la dictadura en el marco del derecho romano clásico pero lo cierto es que ni la regla de los seis meses de ejercicio para todo quien era nombrado dictador se cumplía casi nunca ni tampoco los tradicionales medios de control propios de los tribunos de la plebe como la intercessio, la provocatio ad populum o la Ley Valeriae Horatiae del año 449 fueron aplicados al dictador sino tardíamente dado que, en principio, estos derechos de control sólo podían ejercerse en el pomerium mientras que el dictador no tenía limitaciones ni materiales ni territoriales para su imperium.

Por otra parte, Laurens Janssen ha demostrado que cuando el dictador dejaba su cargo, siempre se utilizaba en los textos el verbo abdicare -que muestra la inexistencia de un límite temporal al ejercicio de un cargo- y nunca su contraparte abire que implica una deposición automática y no voluntaria de una magistratura (JANSSEN, 1960, p. 91-108). A partir de estas características puede verse que la dictadura romana no representaba una violación del orden constitucional puesto que no existía ninguna prohibición respecto de esta institución. Pero tampoco se trataba de una magistratura establecida por medio de un estado de excepción ya que ni el dictador ni el cónsul que lo nombraba suspendían la validez del orden normativo regular. Sin embargo, el dictador sin suspender el orden legal podía situarse en una esfera en cuyo interior se ubica una praxis que es independiente del andamiaje jurídico. Este tipo de acción propia del dictador no puede recibir otro nombre posible que el de gestión de una crisis (SAINT-BONNET, 2001, p. 50-59).

De este modo, el instituto que en el derecho romano realmente se corresponde con el estado de excepción es el iustitium declarado por medio de un

\footnotetext{
2 Una cuestión subsidiaria a nuestro presente análisis pero que merecería la atención por parte de los estudiosos es el hecho de que Carl Friedrich ha sido quien primero facilitó la publicación del estudio de Hannah Arendt sobre la noción jurídico-filosófica de "auctoritas" en la teoría política romana (ARENDT, 2006, p. 91-141). Sobre las relaciones entre las teorías de Friedrich y aquellas de Carl Schmitt, cf. LIETZMANN, 1997, p. 174-194.
} 
senatus consultum ultimum (BARBAGALLO, 1900; DUPLÁ ANSUATEGUI, 1990). Este punto, no siempre claramente distinguido por los investigadores modernos, había sido, no obstante, señalado ya por Maquiavelo quien en sus Discursos evocó la diferencia entre la dictadura y la medida conocida como senatus consultum ultimum: "Aunque después, en los últimos tiempos, los Romanos se habituaron a dar tal poder al Cónsul, y no al Dictador, con estas palabras: "Videat Cónsul, ne Respublica quid detrimenti capiat" (MAQUIAVELO, 2004, p. 141). Por otra parte, además del iustitium, existían otras medidas de la excepción en Roma conocidas como saga sumere, evocatio, crimen maiestatis y declaración de hostis publicus.

Ahora bien, ¿por qué es posible decir que el iustitium es el paradigma del estado de excepción? En uno de los estudios más exhaustivos que existen sobre esta cuestión podemos leer:

cuando el derecho ya no estaba en condiciones de cumplir su deber supremo, que es garantizar el bien común, se abandonaba el derecho por esa oportunidad, y así como en los casos de necesidad los magistrados eran liberados de los vínculos de la ley a través de un senadoconsulto, del mismo modo en el caso más extremo es el derecho el que se debe dejar de lado. En lugar de transgredirlo, cuando se volvía nocivo se lo sacaba del medio, se lo suspendía a través de un iustitium (NISSEN, 1877, p. 99).

Como se puede apreciar, el iustitium es declarado por medio de un senadoconsulto que presupone la presencia de un tumultus. Ahora bien, todas éstas no son categorías del derecho penal, sino del derecho constitucional y marcan el punto en el cual el derecho se suspende frente a la guerra civil. Cicerón recuerda esta institución que, una vez declarada, autorizaba a cualquier ciudadano a dar muerte al insurrecto ante la caducidad del derecho a los fines de la salvación de la institución republicana:

\footnotetext{
En determinada ocasión el senado estableció que el cónsul Lucio Opimio tomara medidas para que la república no sufriera ningún daño. Ni una sola noche se dilató la acción. Gayo Graco, de padre, abuelo y antepasados muy célebres, fue ajusticiado por sospechas de sedición y el ex cónsul Marco Fulvio sufrió muerte junto con sus hijos. Por un decreto senatorial, se confió la República a los cónsules Gayo Mario y Lucio Valerio. ¿Se puede decir, por otra parte, que se les haya aplazado así fuere por un solo día al tribuno de la plebe Lucio Saturnino y al pretor Gayo Servilio la pena de muerte decretada por la república? (CICERON, In Catilinam I, 2, 4).
} 
En todos estos casos, la consecuencia de la proclamación del iustitium por medio de un senadoconsulto conllevaba la declaración del rebelde como hostis. De este modo, al enemigo público "se lo privaba radicalmente de todo status jurídico y por lo tanto podía ser despojado de sus bienes en cualquier momento y llevado a la muerte" (AGAMBEN, 2004, p. 146). Ahora bien, para Agamben este instituto judicial no se limita a explicar un fenómeno histórico sino que resulta ser paradigmático del proceder intrínseco a la estructura de todo el derecho occidental que no puede realizarse en la praxis sin inejecutarse al mismo tiempo (AGAMBEN, 2004, p. 81-83). Desde esta perspectiva, el iustitium no es equiparable a la dictadura puesto que ésta funciona -aún con todos los matices del caso- dentro del esquema del derecho mientras que el primer instituto presupone el carácter absoluto de la anomia y una suspensión del orden jurídico in toto.

En 1996, poco después de la publicación del primer volumen del proyecto Homo sacer de Agamben, los romanistas reaccionaron ante el análisis agambeniano de la "sacralidad" jurídica. El centro de la atención de los romanistas no se refiere tanto al funcionamiento de la pena de la sacralidad en la jurisprudencia romana sino que más bien sus objeciones se dirigen al hecho de que el filósofo italiano habría elegido una figura impropia para la ejemplificación de la excepción como lo es el caso del homo sacer, paradigma de quien podía ser dado de muerte por cualquier ciudadano en el derecho romano arcaico, precisamente, porque se lo apartaba de la esfera del derecho y de las concomitantes prohibiciones de homicidio.

Más allá del caso extremo de algunos especialistas que han considerado demasiado frágil la existencia histórica misma del homo sacer (KIESOW, 2002, p. 62 ), hay estudiosos que consideran a esta última una figura inadecuada para dar cuenta de la excepcionalidad soberana puesto que se trata de un castigo del derecho penal instituido por una condena popular que se habría realizado por fuera de los marcos del poder soberano. Los romanistas señalan que el poder soberano en el derecho romano arcaico castigaba por medio del sacrificium o sea con una pena capital de tipo ritual. En este sentido, el homo sacer quedaría excluido de los medios de la penalización soberana por tratarse de un asesinato consentido a los privati en forma no ritual pero sólo a éstos y no a los magistratus legitimi (FIORI, 1996, p. 443). 
En consecuencia, la única figura que realmente representa la excepción soberana sería la del hostis rei publicae que es privado de todo derecho y asesinado por el poder soberano mediante la declaración de un iustitium con su correspondiente senatus consultum ultimum. Sin embargo, es posible señalar notables analogías entre el homo sacer y el hostis rei publicae, entre las que cabe mencionar el hecho de que, si el culpable estaba en manos del senado, comportaba su inmediata ejecución. Pero, al contrario, si no era posible dar con el culpable inmediatamente, el senatus consultum ultimum consentía, al menos desde el año 88 a. C. que cualquier ciudadano pudiese asesinarlo sin resultar pasible de haber cometido un homicidio. Como corolario de estas características propias del instituto, la declaración de hostis publicus podía también ser emitida sin que fuese necesaria la proclamación de un estado de excepción aún si la acción podía ser concebida como un bellum iustum.

Ciertamente, es posible concebir a la declaración de hostis publicus como una forma de "desacralización" de la consecratio capitis propia de la sacratio originaria. En este sentido, estaríamos frente al mismo proceso que condujo de la consecratio bonorum a la publicatio (SALERNO: 1990). Cabe preguntarse, entonces, el motivo por el cual el propio Agamben no señaló explícitamente las simetrías contundentes que existen entre ambos institutos. Dicho de otro modo, ¿por qué razón Agamben desplaza su atención de la figura del homo sacer como paradigma de la excepción soberana a la del iustitium como forma última de la anomia fundamental del derecho?

El problema resulta vasto pero una de las posibles vías de indagación se encuentra en considerar que, por un lado, implícitamente Agamben reconocía este parentesco profundo entre ambos institutos al declarar que la excepción soberana (presente tanto en el homo sacer como en el iustitium) imposibilita distinguir entre vida y derecho así como entre anomia y nómos (AGAMBEN, 2004, p. 157). Por otro lado, resulta insoslayable tomar en consideración las reformulaciones agambenianas sobre el estado de excepción a partir de sus indagaciones sobre la genealogía de la "teología económica" cristiana medieval que, sobre la base de un proceso de secularización de larga duración, afecta a la praxis gubernamental contemporánea bajo el modelo del managment y la administración de la vida. 
En la genealogía del poder gubernamental moderno, Agamben ha mostrado cómo la noción griega de oikonomía, luego reapropiada por parte de la teología y el derecho romano-canónico medievales, tomó una de sus formas privilegiadas bajo la denominación, precisamente, de "excepción" (AGAMBEN, 2007, p. 63). Esto significa, precisamente, que la oikonomía como administración es consustancial con la suspensión de la fuerza-de-ley. Como señala el filósofo italiano, "el paradigma del gobierno y el del estado de excepción coinciden en la idea de oikonomia, de una praxis de gestión que gobierna el curso de las cosas" (AGAMBEN, 2007, p. 64).

De esta forma, es posible considerar que tanto la institución romana del homo sacer como la del iustitium representan dos declinaciones compatibles de un mismo paradigma, el estado de excepción (llevado a su punto de madurez teórica por la teología política medieval), el cual, al mismo tiempo, entra en una relación complementaria con la oikonomia como gestión biopolítica de la vida en las sociedades globales. De hecho, esta profunda relación complementaria hará que Agamben asimile "la teoría schmittiana del estado de excepción" con el "modelo del poder angélico", esto es, precisamente con el arquetipo teológico de la praxis de gobierno propia de la oikonomía (AGAMBEN, 2007, p. 294). Llegados a este punto, la teología política y la teología económica, la excepción y la administración, se articulan como los dos polos de la máquina gubernamental moderna.

\section{EL PROBLEMA DE LOS DESASTRES ECOSISTÉMICOS. UN ESBOZO GENEALÓGICO}

Tomando en cuenta todos los análisis propuestos por Agamben y que, de modo sucinto, hemos presentado en la sección precedente, querríamos ahora avanzar la hipótesis de que el problema de los desastres ecológicos masivos (en un amplio abanico, desde los desastres naturales hasta las nuevas amenazas nucleares) pueden ser conceptualizados, desde su dimensión política, como formas del ejercicio del poder gubernamental tal y como éste fue concebido en la Modernidad según su origen en la teología económica medieval. Desde luego, este análisis no quita importancia ninguna a otros abordajes desde la filosofía y la teoría política sino que señalan la pertinencia de incorporar a estos debates una perspectiva anclada en la filosofía agambeniana. Esta hipótesis se sustenta en los 
primeros resultados de una genealogía de la noción de "catástrofe" como centro teórico de los dispositivos de gobierno en las sociedades globales de nuestro presente.

De hecho, el vocabulario político de Occidente se ha cimentado, a lo largo de los siglos, según un cuidadoso elenco de nociones. Ante un primer examen, el término "catástrofe" podría parecer ausente de las preocupaciones fundacionales de los grandes clásicos de la filosofía política si tomamos como medida de comparación el peso de nociones tales como soberanía, pueblo o Estado. Es decir que la gramática política occidental, al menos en su superficie más visible, ha podido construir sus edificios teóricos sin colocar a la vista el problema de las catástrofes naturales.

Una de las tantas razones para esta ausencia podría buscarse, precisamente, en los fines últimos que la política se había dado a sí misma, al menos, desde su comienzo griego. Si dirigimos nuestra atención al complejo mosaico de la filosofía política griega, una inveterada fórmula destinada a volverse canónica llamará inmediatamente nuestra atención. En efecto, consideremos el programa que Aristóteles diseña en su Política y que, innegablemente, ha marcado durablemente (aún si de modos muchas veces contradictorios) el pensamiento político de Occidente: "la ciudad [...] surgió por causa de las necesidades de la vida, pero existe ahora para vivir bien (eu zên)" (ARISTÓTELES, Política, 1252b, 30).

La fórmula ha sido comentada y glosada con encomiable meticulosidad y las consecuencias que de ella se derivan han sido escrutadas con empeño inigualable a lo largo de los siglos. Sin embargo, por debajo de la masa documental que constituye el edificio de la ciencia política, el problema de la catástrofe no ha cesado de acechar la promesa de felicidad y de buen vivir que la política se había dado a sí misma como tarea desde sus inicios griegos.

Si habremos de estudiar el fenómeno de la catástrofe, entonces, se impone una reflexión sobre el término mismo. En griego antiguo, katastrophé tiene una significación técnica muy precisa que no está en absoluto vinculada con los desastres naturales (o civilizacionales) que son connotados por el sentido moderno del vocablo. Al contrario, su campo semántico recubre el espacio del teatro y, más específicamente, designa la conclusión o el cierre dramático de una pieza teatral. El comentario de Evantio (así como el de Donatio) resulta de extrema claridad al 
respecto. La catástrofe, en esta perspectiva, como dispositivo retórico de cierre de la pieza teatral tiene la capacidad de ofrecer el sentido último al conjunto dramático desarrollado en su transcurso (EUANTHIUS, De fabula hoc est de comoedia, IV, 5).

En otras connotaciones del término, será posible hallar la mención de la "catástrofe" como el fin o conclusión de una vida, tal y como nos sirve de testimonio la expresión katastrophé toû zên (MENANDRO, Perikeiromene, 12). Desde luego, los griegos tenían otros vocablos para designar lo que hoy entendemos por catástrofes naturales, preferencialmente el término "cataclismo (kataklismós)"3 que, por ejemplo, podemos encontrar en Platón asociado a la inundación (PLATÓN, Leyes, 679d). No obstante, la historia lexicográfica del término "catástrofe" que tiene su origen en la esfera del teatro para acabar designando el significante supremo del desastre último merece atención porque revela, precisamente, tanto una mutación epistemológica de magnitud cuanto permite entrever una genealogía de las formas y el ejercicio del poder político.

Por otra parte, también es elocuente la duplicidad del término "cataclismo" dentro del ámbito del griego antiguo puesto que no sólo hace referencia a los desastres naturales sino que, progresivamente y del mismo modo que con los siglos ocurrirá con el término "catástrofe", sufre un desplazamiento hacia la esfera de la política. De este modo, cuando Demóstenes pronuncia, en el 330 a.C. su alocución en defensa de Ctesifonte, podemos ver una utilización política del término cataclismo cuando hace uso de la expresión "kataklismòn tôn pragmáton" (DEMÓSTENES. Sobre la corona, 214). En este punto, Demóstenes ya anticipa los usos que tendrá el "catastrofismo" en los discursos políticos de Occidente y que hoy se tornan omnipresentes para hacer referencia a las catástrofes tanto políticas como económicas. La ideal filosófico de la felicidad se ve, desde el inicio mismo de la politicidad occidental, sacudido desde sus bases por el cataclismo de la praxis que no permite la articulación armoniosa soñada por la tradición.

La Modernidad no utilizará inmediatamente el término "catástrofe" para referirse a los desastres naturales que han condicionado durablemente los pliegues de la conciencia europea a partir del siglo XIV en adelante. En muchos casos suele citarse, como ejemplo y punto de partida de la reflexión filosófica sobre el desastre,

\footnotetext{
${ }^{3}$ Como puede notarse, la connotación filológica del prefijo "katá" se torna indispensable. En este sentido, cf. BENVENISTE, 1935.
} 
al terremoto de Lisboa de 1755 (BREIDERT, 1994). Sin embargo, resulta decisivo no olvidar un antecedente primordial aunque menos conocido que es el terremoto de Chile de 1647 que ya había impactado sobre la reflexión filosófico-literaria del continente europeo. Un ejemplo de magnitud, en este ámbito, está constituido por el texto de Heinrich von Kleist sobre el particular en el cual todavía no encontramos el término "catástrofe" aplicado al terremoto sino que el escritor decanta su preferencia por "ruina" o "perdición" (Verderben) (KLEIST, 1978, p. 159). ${ }^{4}$

De todos modos, si llevamos nuestra atención a la conceptualización del terremoto de Lisboa por parte de algunos de los filósofos más importantes de la Era Moderna (HAMACHER, 1999, p. 261-293), podremos constatar, por ejemplo, que Voltaire tampoco utiliza el vocabulario de la catástrofe sino el del desastre natural (VOLTAIRE, 2003, p. 219-220) pensado como mal moral y físico que sólo puede ser concebido según los esquemas de una sutil teología providencial (BESTERMAN, 1956, p. 7-24).

Esta herencia como marco conceptual estará asimismo presente en la tratadística kantiana sobre el terremoto de Lisboa aún si en el caso del filósofo alemán es posible entrever una especulación digna de una proto-sismología (KANT, 1902, vol. I, p. 429-462). En todos los casos, el ecosistema en cuanto tal como acontecer de una historia natural no es imaginable, en última instancia, sino bajo el registro del mal teológico y mediante una figuración antropológica donde el destino del hombre se torna en objeto de la reflexión filosófica.

Por lo tanto, la conceptualización del desastre natural como "catástrofe" será un poco más tardía en la filosofía y, aún en una época donde su uso estaba ya plenamente establecido, podremos encontrar autores que no se sirven de este vocablo para dar cuenta de los cataclismos civilizacionales. Un ejemplo de esta tendencia lo brinda Oswald Spengler cuando escribe: "toda cultura (Kultur), toda época primitiva (Frühzeit), todo florecimiento (Aufstieg) y decadencia (Niedergang), y cada una de sus fases y períodos necesarios, posee una duración fija, siempre la misma, que se reitera siempre con la insistencia de un símbolo (mit dem Nachdruch eines Symbols)" (SPENGLER, 1963, p. 147). ${ }^{5}$

\footnotetext{
${ }^{4}$ Para una exégesis de este texto, resulta fundamental remitirse a SCHRADER, 1991, p. 27-42 así como también SCHÜTTE, 2006, pp. 97-110.

${ }^{5}$ Para un análisis del contexto cultural donde surge la obra de Spengler, cf. RINGER, 1969.
} 
El espacio científico-especulativo propio de la Modernidad francesa será decisivo en la mutación de los sentidos del término "catástrofe" y, por lo tanto, debemos ahora circunscribirnos a este ámbito geográfico-cultural. En principio, podemos hallar en este contexto una situación similar a la que encontramos precedentemente en esta breve pesquisa. Una aproximación fundamental nos la brinda, una vez más, la lexicografía que se cultiva en Francia hacia finales del siglo XVII.

Con este propósito como guía, más aún que a la primera e insuficiente edición del diccionario de la Academia francesa de 1694, conviene referirse a la obra maestra de la lexicografía del período como es el Diccionario de Antoine Furetière. Allí podemos dar con la siguiente definición: "catástrofe: término de la poesía. Son el cambio y la revolución que tienen lugar en un poema dramático y que, de modo ordinario, constituyen su término" (FURETIĖRE, 1690, s.v. "catastrophe"). Al mismo tiempo, el término en su connotación griega se extiende de modo figurado, por ejemplo, para dar cuenta del final funesto e infeliz de una vida.

Sin embargo, la era moderna marcaría un nuevo espesor del término. Una perspectiva arqueológica muestra que el término comienza en la paleobiología para trasladarse luego al escenario de la política. En este pasaje semántico que enmarca una auténtica mutación epistemológica, la obra de Georges Cuvier resulta un hito de la máxima trascendencia. Para el naturalista,

La vida ha sido a menudo perturbada en esta tierra por acontecimientos terribles; calamidades que, en los inicios, han quizá removido en gran escala, la apariencia entera del planeta, pero que desde entonces son siempre menos profundas y menos generales. Innumerables seres vivientes han sido víctimas de estas catástrofes (CUVIER, 1812, volumen 1, p. 11). ${ }^{6}$

El problema de la "discontinuidad de las formas vivas" que se plantea con Cuvier presupone una ontología propia de la época clásica que ha sido analizada por Michel Foucault. En la obra de Cuvier halla su lugar de manera preferencial el cuasi-trascendental de la Vida analizado en la arqueología foucaultiana y que tomará simplemente otro nombre cuando sea pronunciado con la cadencia distintiva de las relaciones de poder: se trata de la biopolítica.

${ }^{6}$ Para la teoría de Cuvier y su debate con Étienne Geoffroy Saint-Hilaire, cf. APPEL, 1987, p. 202237. 
Como prueba de ello, baste referirnos a la caracterización que Foucault realiza del problema de la vida, a partir de la obra de Cuvier, en su arqueología de la episteme de la época clásica:

[la vida] se revela mortífera en el mismo movimiento que la destina a la muerte. Ella mata porque vive. La naturaleza ya no puede ser bondadosa [...] la vida no puede ya ser separada de la muerte, la naturaleza del mal, ni los deseos de la contra-naturaleza (FOUCAULT, 1966, p. 290).

La paleontología de Cuvier eleva al rango de teoría científica todo cuanto el terremoto de Lisboa había ya anticipado bajo el ropaje de la teología económica cuya genealogía ha llevado adelante Agamben oportunamente. A partir de ese momento, la naturaleza pasará a formar parte de un medio hostil cuya existencia supera en una temporalidad de millones de años la aparición del hombre sobre la Tierra. La catástrofe será entonces el lenguaje más propio en que la naturaleza expresará su indiferencia antropológica ante la presencia del hombre en su seno. Sus movimientos geológicos, sus alteraciones y sus catástrofes se producirán sin que el hombre pueda evitarlas.

El nuevo escenario espistemológico, no obstante, supondrá una lección para el nuevo orden de los discursos y la praxis del poder en la Modernidad. El "catastrofismo" abre las puertas a una nueva geohistoria de la Tierra y, de modo concomitante, el biopoder hará suya una concepción en la cual el desastre no será ya un acontecimiento que deba ser evitado (puesto que se revela ineluctable) sino, al contrario, gobernado en sus efectos una vez estos desencadenados.

En su curso del Collège de France de 1977-1978, Foucault definirá los rasgos propios de la racionalidad gubernamental de la biopolítica moderna a través del caso de los fisiócratas y su concepción de los "acontecimientos-calamidades":

\footnotetext{
En el fondo, la calamidad, la carestía, tal y como se la concebía hasta ese momento, era a la vez un fenómeno individual y colectivo, y de esta manera había gente que tenía hambre, poblaciones enteras que tenían hambre, la nación tenía hambre, y era eso precisamente, esta especie de solidaridad inmediata, de masividad del acontecimiento, lo que constituía su carácter de calamidad (FOUCAULT, 2004, p. 43).
} 
Lo importante, entonces, no será impedir la calamidad sino dejar que se produzca y administrarla para que, en tanto fenómeno, alcance por sí misma su punto de autorregulación:

De tal suerte que no habrá más carestía en general a condición de que la
haya para toda una serie de gente, en toda una serie de mercados [...]. El
acontecimiento-carestía resulta así disociado. La carestía-calamidad
desaparece, pero la rarefacción que hace morir a los individuos no
solamente no desaparece sino que no debe desaparecer (FOUCAULT,
2004, p. 43).

En otras palabras, del "catastrofismo" teológico y científico se podrá pasar a una analogía discursiva en el campo político que hace derivar de la calamidad un carácter necesario cuya implicación será que todo desastre (sin distinción entre su forma natural, social o cultural) no sólo será permitido sino también, en muchos casos, inducido so pretexto de un beneficio para comunidad política transformada ahora en "población".

Esta imbricación entre la oikonomía teológica estudiada por Agamben para el período medieval y su secularización moderna en la conceptualización científica de las "catástrofes naturales" puede encontrar un punto genealógico fundamental en la teodicea de Leibniz cuando el filósofo se pregunta por el sentido de "calamidades, diluvios e inundaciones" para concluir que son el producto dirigido de una ratio gubernatoria divina: "los desórdenes ocurrieron dentro del orden" y, en razón de ello, han sido "regulados" por acción de la oikonomía teológica (LEIBNIZ, 2013, p. 259).

Desde este punto de vista, no debe sorprendernos que la lexicografía acompañe este proceso de mutación en el orden del poder. Así, noción de catástrofe adquiere una nueva significación, inspirada por los nuevos descubrimientos en paleontología así como por los cambios en la concepción política del gobierno de las cosas y de los hombres. De allí que Émile Littré en su diccionario históricoetimológico pueda llegar a invertir el sentido originario de catástrofe como conclusión de una pieza teatral para privilegiar la acepción de "gran infelicidad" y dar como ejemplo, siempre traído a colación pues tiene el eco del terremoto de 1755 en Lisboa, que reza "aquel terremoto fue una catástrofe horrorosa (épouvantable catastrophe)" (LITTRÉ, 1872-1877, s.v. "catastrophe”). 
Esto significa que ninguna catástrofe es enteramente "natural" y que, por tanto, siempre resulta posible una semiótica social del fenómeno. Su inscripción dentro de un tejido de significaciones sociales permite su introyección histórica y su interpretación cultural. En el caso de las sociedades históricas, muchas veces el testimonio arqueológico es la única forma de acceso a los restos de un desastre ecosistémico.

Ahora bien, en nuestra época, los progresos de la técnica han hecho posible, por primera vez en la historia, no ya que los hombres actúen de manera tal que puedan cambiar la biosfera (situación presente desde los inicios mismos de la vida social entre los humanos) sino que, más dramáticamente, puedan destruirla y destruirse a sí mismos mediante una catástrofe autoinducida. El paradigma de este último tipo de catástrofes ha sido (y continúa siendo) la bomba atómica.

Uno de los estudios más contundentes en esta dirección ha sido escrito por Karl Jaspers e injustamente olvidado por la teoría política. La inquietud por la catástrofe que la tecnología atómica puede producir es la interrogación por la catástrofe hiperbólica, aquella que pone en jaque la propia supervivencia de la Humanidad. Por tanto, para Jaspers se trata de una inquisición que sólo puede abordarse como el horizonte mismo de nuestra existencia. De allí que Jaspers denuncie la posición que se niega a pensar el problema del desastre último:

\footnotetext{
Sobre todo, nada se desea saber del peligro atómico. Se formulan evasivas: ante la amenaza de una catástrofe total no se puede hacer ni política ni planificación. Queremos vivir, no morir. Pero de producirse ese cataclismo, todo habrá terminado para nosotros. Afírmese que no tiene sentido pensar en ello [...] pues se arriesga tornar insoportable la vida. Pero precisamente por ello puede promover la transformación que por sí sola modifique esa situación real tan amenazante (JASPERS, 1961, p. 19).
}

Desde la perspectiva de Jaspers, el tono apocalíptico que puede tener en la reflexión filosófica la conceptualización de la catástrofe, lejos de alejar a la comunidad de los hombres de la política, al contrario, los incita a tomar parte de una acción politizada precisamente sobre la base del horizonte último que, sobre toda filosofía política, introduce el problema de la catástrofe total. Más aún, como señala Jaspers, esta alarma apocalíptica es "la única oportunidad de una renovación política que elimine el peligro de una catástrofe" (JASPERS, 1961, p. 20). 
En esta perspectiva, una genealogía del desastre ecosistémico en la política occidental no puede evitar recordar la peste de Atenas, una de las catástrofes más mortíferas en la historia del mundo griego antiguo que se desató en el segundo año de la guerra peloponesia, hacia el 430 a.C. El relato histórico más fecundo que tenemos de las modalidades de la peste ${ }^{7}$ y de sus efectos inmediatos sobre la forma de vida de la ciudad de Atenas corresponde a Tucídides. ${ }^{8}$ La peste comenzó en Etiopía y fue recorriendo diversas zonas geográficas hasta entrar en Atenas a través del Pireo. La descripción de Tucídides de la fenomenología social de la plaga sigue un patrón que había sido ya establecido por Homero en la llíada. El relato tiene su punto de partida, entonces, en el efecto de la enfermedad sobre los animales:

\begin{abstract}
Las aves y cuadrúpedos que comen carne humana, a pasar de las muchas personas que habían insepultas, o no se acercaban o perecían tras probarlas; una prueba: hubo una clara desaparición de tal clase de aves y no se las veía ni en las circunstancias mencionadas ni en otras; los perros se prestaban más a la observación de lo sucedido por su convivencia con el hombre (TUCÍDIDES. Historia de la Guerra del Peloponeso. II, 50).
\end{abstract}

Justamente, entre los ciudadanos, el efecto fue devastador. Había quienes optaban por abandonar a sus enfermos así como otros morían por el contagio producido en el cuidado de los afectados. La piedad religiosa se quebró debido a que "derrotados por la extensión de la enfermedad, se cansaron de hacer las lamentaciones por los que morían" (TUCÍDIDES. Historia de la Guerra del Peloponeso. II, 51). En directa relación con este aspecto, las prácticas de la sepultura se vieron completamente subvertidas (PARKER, 1983):

\begin{abstract}
Muchos se prestaron a entierros indecorosos ante la falta de lo preciso por los continuos entierros efectuados previamente; unos tras poner su muerto en piras ajenas, anticipándose a los que las habían amontonado, prendían fuego, y otros, mientras ardían otros cadáveres, echaban encima el que llevaban y se marchaban (TUCíDIDES. Historia de la Guerra del Peloponeso. II, 51,6).
\end{abstract}

A este proceso de resquebrajamiento de los tejidos simbólicos de la unidad religiosa de la ciudad le siguió un proceso de colapso absoluto del sistema políticoeconómico en su conjunto:

7 Sobre los conocimientos médicos de Tucídides, elocuentes en su descripción de la peste, cf. RECHENAUER, 1991.

${ }^{8}$ Respecto de Tucídides y el relato de la guerra, cf. CAWKWELL, 1997. 
También en los demás aspectos la enfermedad fue para la ciudad el inicio de la anomia (anomías) [...] Ni el temor de los dioses ni la ley de los hombres (theôn dè phóbos he anthrópon nómos) eran un obstáculo, por juzgar que lo mismo daba ser respetuoso que no, cuando veían que todos perecían por igual y por creer que nadie viviría hasta el juicio para pagar por su delitos sino que ya pendía sobre ellos y estaba decretado un castigo mucho mayor $y$, antes de que les cayese encima, era natural que disfrutasen algo de la vida (TUCÍDIDES. Historia de la Guerra del Peloponeso. II, 53, 4).

La expresión utilizada por Tucídides no deja lugar a duda: la peste precipita a la ciudad en la anomia radical (ORRÙ, 1987). En efecto, estos textos dan cuenta de un tipo de anomia que, según Agamben, habita en el dispositivo mismo que permite todo ordenamiento jurídico. Aquí la ciudad no sólo es pensada tanquam dissoluta sino que, además, se halla histórica y efectivamente diezmada.

De hecho, podría sostenerse que esta peste es la primera que ha tenido un auténtico "efecto filosófico" sobre la historia del pensamiento: ha permeado las concepciones políticas de Tucídides así como ha sido determinante para la filosofía política de la catástrofe moderna (GINZBURG, 2008). Los Tiempos Modernos, de hecho, no se han constituido sino a partir de una sucesiva escalada de catástrofes derivadas de las pestes que se produjeron desde los tiempos de la Europa medieval. El Renacimiento mismo se inaugura con una brutal peste sobre Florencia, uno de cuyos relatos más agudos se encuentra en el Decamerón de Giovanni Boccaccio.

En efecto, se trató de una peste tan mortífera que, encontrando un eco en el relato de Tucídides, Boccaccio pudo escribir que "en medio de tanta aflicción y miseria, la venerable autoridad de las leyes, tanto divinas como humanas, había caído o estaba disuelta, sin el amparo de sus ministros y ejecutores, los cuales estaban, como todos los demás hombres, muertos o enfermos" (BOCCACCIO, 1956, p. 8). Con todo, esta peste fue originada y difundida a partir del comercio protoglobal que la nueva era traía consigo. Como ha sido señalado oportunamente por Peter Sloterdijk:

Desde 1348 saben los europeos que las grandes ciudades comerciales son espacios de infección. Constituyen zonas de riesgo [...] sus habitantes han de comprender ahora, en un proceso de aprendizaje pavoroso, que las riquezas y las infecciones viajan juntas[...] Casi se podría decir que Europa se convierte desde entonces en un espacio experimental en el que se somete a prueba la unidad y diferencia entre lo salutífero y lo mortífero (SLOTERDIJK, 2013, p. 20-21). 
Si el filósofo alemán lleva razón y los florentinos fueron quienes primero debieron experimentar el nuevo orden de la globalización en la Modernidad temprana, no menos cierto es que, a partir de entonces, las catástrofes no cesaron de producirse y de conceptualizarse de manera cada vez más acuciante. Al mismo tiempo, los descubrimientos de la ciencia moderna nos han colocado sobre el abismo de nuevos tipos de catástrofes técnicas que no habían podido ser consideradas hasta el presente.

\section{CONCLUSIONES: EL DESASTRE TÉCNICO-AMBIENTAL Y EL PROBLEMA POLÍTICO DE LA EXTINCIÓN}

En los tiempos contemporáneos, la capacidad de destrucción significativa, potencialmente irreparable e, incluso, absoluta del ecosistema terrestre se presenta no ya como una posibilidad teórica sino también como una circunstancia sociohistórica ineludible. Desde esta perspectiva, Günther Anders ha señalado agudamente que una nueva cartografía epocal se dibuja bajo nuestra mirada en tanto y en cuanto debemos pensar "nuestra existencia bajo el signo de la bomba (unser Dasein unter dem Zeichen der Bombe)" (ANDERS, 1961, p. 235). Esta situación conlleva el hecho fundamental de que el hombre ha dejado de ser el fundamento mismo de la historicidad. En el estado presente de situación, la técnica es el verdadero sujeto de la Historia.

Fruto de esta constatación, Anders puede diagnosticar desplazamientos metafísico-políticos de primera magnitud. En primer lugar, las sempiternas "preguntas sobre el cómo (Wie-Fragen)" son ahora reemplazadas por una única preocupación: "la pregunta por el si (Ob-Frage)" la humanidad podrá subsistir o no sobre el planeta. "Ceguera ante el apocalipsis (Apokalypse-Blindheit)" es el modo según el cual Anders califica la actitud de quienes, por temor o pereza teórica, se niegan a abordar esta cuestión en su plena magnitud (ANDERS, 1961, p. 238). Ciertamente, no se trata aquí de un apocalipsis teológico sino, al contrario, de una profunda mutación histórica puesto que los "señores del apocalipsis (Herren der Apokalypse)" ahora somos los habitantes del planeta que, como característica distintiva, poseemos una inusitada potestas annihilationis (ANDERS, 1961, p. 239). 
Al mismo tiempo, si es posible recordar aquel silogismo escolásticouniversitario que siempre ha definido el espíritu humanista de la filosofía bajo la conclusión de que "todos los hombres son mortales", hoy en día un sustituto se ha presentado como huésped ingrato de los nuevos tiempos: "la humanidad (Menschheit) como conjunto es eliminable" (ANDERS, 1961, p. 242). En contrapartida, el eco de la tesis salomónica "todo será pasado (Alles wird gewesen sein)" que resuena desde el fondo de la historia, podrá ser reemplazado por el nuevo murmullo amenazante: "nada habrá existido (Nichts ist gewesen)" (ANDERS, 1961, p. 243).

Aún en el marco de este diagnóstico de una hecatombe desencadenada por la acción humana, Anders toma en cuenta el tipo de catástrofe cuyo esbozo genealógico en la política occidental hemos querido seguir en estas páginas. La desaparición de la Atlántida, si hubiéramos de otorgar algún crédito a la leyenda, señala Anders, no fue únicamente una catástrofe histórica (geschichtliche Katastrophe) sino una de las formas de las catástrofes naturales (Naturkatastrophen). No se trata, en este sentido, de una catástrofe que tiene lugar en el transcurso de la historia sino, al contrario, de un tipo de acontecimiento radical que representa el auténtico "fin de la historia" (ANDERS, 1961, p. 263).

Aún así, las catástrofes de nuevo cuño combinan precisamente elementos de las precedentes generando cataclismos propios de una hibridación entre naturaleza e historia que, como diría Agamben, torna indistinguible la diferencia entre nómos y physis. ${ }^{9}$ Es el caso del desastre de Fukushima cuando, el 11 de marzo del 2011, la susodicha central nuclear fue golpeada por un tsunami produciendo una de las devastaciones ecosistémicas más notorias del presente siglo. Como ha sido señalado,

la catástrofe atómica continúa siendo la catástrofe tendencialmente irremediable cuyos efectos se propagan a través de las generaciones, a través de los suelos y de todas las especies vivientes y de la organización a gran escala de la producción de energía y, por tanto, de su consumo (NANCY, 2012, p. 11).

${ }^{9}$ La situación, por tanto, es distinta de la analizada, para el caso de la Segunda Guerra Mundial, por SEBALD, 2001. 
La taxonomía híbrida del caso es señalada por Nancy: "un sismo y el tsunami que engendra devienen catástrofe técnica, que deviene a su vez sismo social, económico, político y finalmente filosófico" (NANCY, 2012, p. 56). La progresión geométrica del desastre hace pensar a Nancy que no existen más (o no sólo) catástrofes completamente naturales y que sólo tienen lugar catástrofes civilizaciones a gran escala ya que el hombre ha transformado duraderamente su relación con la naturaleza.

Ante este panorama, no es extraño entonces constatar que Bruno Latour subraye cómo nuestra época actual ha entrado en un régimen histórico comparable a la era de Cristóbal Colón salvo que, esta vez, no se trata para los europeos de percatarse de la existencia de un Nuevo Mundo sino, para la humanidad toda, se presenta el desafío de habitar no sólo el "Viejo Mundo" (como escribe Latour) sino el globo en su totalidad sobre bases enteramente nuevas. Así Latour propondrá el camino teórico hacia una teología política de la naturaleza que, paradójicamente, hace del nombre "Gaia" el operador que permite pensar, por fin, al ecosistema libre de toda determinación teológica, emergiendo como la gran nueva figura secular de la teoría política (LATOUR, 2015).

De este modo, es posible retomar el camino de una geohistoria y allí resulta inevitable reencontrarse con el "catastrofismo" de Cuvier. En efecto, el paleontólogo francés había podido identificar las épocas "catastróficas" de la historia geológica de la Tierra al demostrar cómo los huesos del mamut eran diferentes de los del elefante moderno. La conclusión a la que llegó Cuvier fue que la especie mamut había sufrido una extinción. La escuela catastrofista, tenazmente combatida por Charles Lyell y Charles Darwin, logró recuperar bríos y demostrar cómo la historia de la Tierra está marcada por convulsiones que ponen a la vida al borde de la extinción. Los geopaleontólogos hablan así de las cinco grandes crisis bióticas en las que desaparecieron por lo menos el 65 por cierto de las especies en un lapso geológico breve. Ahora bien, en la catástrofe masiva del período pérmico, se calcula que despareció más del 95 por ciento de las especies animales marinas.

De este modo, la perspectiva que considera una Sexta Extinción como una próxima catástrofe a considerar se torna de una urgencia determinante. De hecho, es ineluctable que el Homo sapiens concluya su existencia terrestre según las leyes de la geohistoria como resultado de una nueva catástrofe geológica: "si alguna 
certeza podemos inferir del conocimiento del flujo de la vida y de las fuerzas que lo forman es que llegará el día en que pereceremos todos, nosotros y nuestros descendientes" (LEAKEY; LEWIN, 1995, p. 251). A partir de esta constatación, una serie de observaciones se imponen acerca del propio desenvolvimiento del Homo sapiens en el ecosistema terrestre.

Los paleo-biólogos han demostrado cómo, hace aproximadamente 42.000 años, el Homo sapiens migró desde su origen en el este de África hacia la zona geográfica que hoy conocemos como el Asia. Cuando llegó allí, no obstante, encontró que no estaba solo. El territorio se hallaba ocupado por una comunidad, numéricamente mayor, de los llamados hombres de Neandertal. Hoy parece un hecho casi indiscutible para los expertos que ambos géneros de mamíferos hombres cohabitaron juntos durante milenios, llegando incluso a producirse una hibridación genética de la que somos aún herederos.

Sin embargo, en cierto momento, los hombres de Neandertal desaparecieron de la faz de la tierra. Los especialistas aún siguen debatiendo la razón por la cual, hace 30.000 o 25.000 años atrás, sus últimos especímenes abandonaron toda presencia en el ecosistema terrestre sin dejar rastro alguno. Aunque no existe ningún acuerdo establecido al respecto y muchos piensan que, tal vez, el cambio climático fue la causa de su desaparición, a pesar de la enorme capacidad de resistencia de esta línea evolutiva humana a las inclemencias de este tipo (STRINGER, 2001, p. 791-792), una de las teorías -tal vez la más inquietantesostiene que, sencillamente, los neandertales fueron exterminados por los miembros del género Homo sapiens. Sin embargo, aún si este evento sólo fuera sino una especie de mitomotor paleontológico, una vez más, se muestra con toda claridad que el hombre piensa su primer acto político fundacional de toma de la tierra, como un acto eugenésico-social. El nómos de la tierra es también biopolítico por naturaleza.

Una vez liberado el espacio, el animal humano conquistó enteramente el ecosistema hasta causar la desaparición completa de muchas de las especies de mamíferos de las que se alimentaba como cazador-recolector. Esta catástrofe ecosistémica -sin duda, primer acto probado de exterminio de otras especies por parte del hombre- condujo al desarrollo de una primera técnica de intervención artificial sobre el espacio natural: la agricultura y, con ella, al alba de los 
asentamientos humanos que darían inicio a una historia que aún estamos tratando de descifrar. Cuando el hombre se hizo cazador, produjo la aniquilación de especies enteras y, cuando se hizo agricultor, cambió irreversiblemente el ecosistema planetario hasta llegar, inexorablemente, por el aumento exponencial de las antropotecnologías y de la técnica tout court, al borde del abismo en que la especie se encuentra hoy respecto de su capacidad de arrasar completamente la Lichtung que alguna vez le dio origen.

En 1955, Claude Lévi-Strauss publicó Tristes Trópicos, acaso uno de sus libros más lúcidamente nostálgicos y pesimistas (HARTOG, 2005, p. 11-22). A lo largo de laboriosas páginas, el antropólogo va recordando sus viajes y constituyendo una auténtica filosofía del devenir de la especie humana, y de la tarea misma del antropólogo. Sus conclusiones no son precisamente alentadoras para quienes reivindican las potencialidades de la expansión indefinida del Homo sapiens: "el mundo comenzó sin el hombre y terminará sin él. Las instituciones, las costumbres y los usos, que yo habré inventariado en el transcurso de mi vida, son la eflorescencia pasajera de una creación en relación con la cual quizá no posean otro sentido que el de permitir a la humanidad cumplir allí su papel” (LEVI-STRAUSS, 1988, p. 465).

En este punto, Lévi-Strauss hace suya una teoría histórica donde la física ecosistémica penetra completamente dentro de los espacios tradicionalmente definidos como culturales. Desde luego, esta ley gobierna todo el mundo de las creaciones humanas:

\footnotetext{
Sin duda ha construido ciudades y ha cultivado campos; pero, cuando se piensa en ello, esas realizaciones son máquinas destinadas a producir inercia a un ritmo y en una proporción infinitamente más elevados que la cantidad de organización que implican. En cuanto a las creaciones del espíritu humano, su sentido sólo existe en relación con éste y se confundirán en el desorden cuando hayan desaparecido (LEVI-STRAUSS, 1988, p. 466).
}

Universo y mundo humano no son sino un continuum asociado por la misma ley cósmica de entropía irreversible. Al punto tal llega esta convicción de LéviStrauss que en un momento puede declarar: "antes que 'antropología' habría que escribir 'entropología' como nombre de una disciplina dedicada a estudiar este proceso de desintegración en sus manifestaciones más elevadas" (LEVI-STRAUSS, 1988, p. 467). 
En todo caso, el desafío que la catástrofe como extinción masiva lega a la filosofía política por venir es pensar no sólo las causas y consecuencias de las catástrofes que el hombre está en condiciones de inducir a partir de la tecnocracia hipermoderna sino también las catástrofes de orden natural que pueden ir, desde un punto de vista geológico, desde una escala modesta hasta una masividad capaz de provocar una extinción definitiva de la vida. Esto implica pensar sobre bases completamente nuevas el ecosistema terrestre y su inserción en un cosmos que no habrá de ser indulgente con la vida presente en la Tierra.

Este horizonte último con el cual debe medirse el viviente humano obliga a considerar al tríptico de oikonomía-excepción-catástrofe como el centro de la ecoteología secularizada que permea el ejercicio de la política en el mundo contemporáneo. De esta manera, los paradigmas políticos de la peste (Tucídides) y de la catástrofe natural (Cuvier) se han articulado con la teoría del estado de excepción en tanto forma privilegiada de la gubernamentalidad moderna. Desde esa perspectiva, hemos querido sostener la hipótesis de que la arqueología agambeniana de la oikonomía teológica resulta compatible con una genealogía política de los desastres ecosistémicos. Se abre entonces, de manera concomitante, la posibilidad de concebir una ontología por venir (AGAMBEN, 2014, p. 192-227) que sea capaz de colocarse más allá de la distinción entre las esferas de aquello que, desde los antiguos griegos, se ha señalado como el mundo nomotético de la cultura en su tensión con la physis o la naturaleza de cuyo éx-tasis la antropogénesis (AGAMBEN, 2002) ha querido dar cuenta bajo la forma de la política humana.

\section{REFERENCIAS}

AGAMBEN, Giorgio. L'aperto. L'uomo e l'animale. Torino: Bollati Boringhieri, 2002.

Estado de excepción. Homo sacer, II, 1. Traducción de Flavia e Ivana Costa. Buenos Aires: Adriana Hidalgo, 2004.

. Il Regno e la Gloria. Per una genealogia teologica dell'economia e del governo. Homo sacer II, 2. Vicenza: Neri Pozza, 2007.

. L'uso dei corpi. Homo sacer, IV, 2. Vicenza: Neri Pozza, 2014. 
ANDERS, Günther. Die Antiquiertheit des Menschen. Über die Seele im Zeitalter der zweiten industriellen Revolution. München: Verlag C.H. Beck, 1961.

APPEL, Toby. The Cuvier-Geoffroy Debate. French Biology in the Decades before Darwin. Oxford: Oxford University Press, 1987.

ARENDT, Hannah. Between Past and Future. New York: Penguin Books, 2006.

ARISTÓTELES. Politics. Edición de William David Ross. Oxford: Clarendon Press, 1957.

BARBAGALLO, Corrado. Una misura eccezionale dei romani. Senatus-

Consultum Ultimum. Studio di storia e di diritto pubblico romano. Roma: E. Loescher, 1900.

BENVENISTE, Émile. Origines de la formation des noms en indo-européen. Paris: Maisonneuve, 1935.

BESTERMAN, Theodore. Voltaire et le désastre de Lisbonne: ou, La mort de l'optimisme. Studies on Voltaire and the Eighteenth Century, n. 2, p. 7-24, 1956.

BIGO, Didier. Sécurité et inmigration: vers une gouvernamentalité par l'inquiétude? Cultures \& Conflicts, n. 31-32, p. 13-38, 1998.

BOCCACCIO, Giovanni. Decameron. Edición italiana de Vittore Branca, Torino: Utet, 1956.

BREIDERT, Wolfgang. Die Erschütterung der volkommenen Welt. Die Wirkung des Erdbebens von Lissabon im Spiegel europäischer Zeitgenossen. Darmstadt: Wissenschaftliche Buchgesellschaft, 1994.

CAWKWELL, George. Thucydides and the Peloponesian War. London: Routledge, 1997.

CICERÓN. Discours. Catilinaires. Henri Bornecque (Ed.). Paris: Les Belles Lettres, 1926.

CUVIER, Georges. Recherches sur les ossemens fossiles de quadrupèdes, où l'on rétablit les caractères de plusieurs espèces d'animaux que les révolutions du globe paroissent avoir détruites. Paris: Deterville, 1812. 4 v.

DEMÓSTENES. Orationes. Edición de Samuel Henry Butcher, Oxford: Clarendon Press. 1903.

DUPLÁ ANSUATEGUI, Antonio. Videant Consules. Las medidas de excepción en la crisis de la República romana. Zaragoza: Universidad de Zaragoza, 1990. 
EUANTHIUS. De fabula hoc est de comoedia. (In) KAIBEL, Georg. Comicorum Graecorum Fragmenta. Voluminis I, fasciculus prior. Berlin: apud Weidmannos, 1899.

FIORI, Roberto. Homo sacer. Dinamica politico-costituzionale di una sanzione giuridico-religiosa. Napoli: Jovene Editore, 1996.

FOUCAULT, Michel. Les mots et les choses. Une archéologie des sciences humaines. Paris: Gallimard, 1966.

FOUCAULT, Michel. Sécurité, Territoire, Population. Cours au Collège de France 1977-1978, Paris: Gallimard-Seuil, 2005.

FURETIÈRE, Antoine. Dictionaire universel contenant generalement tous les mots françois tant vieux que modernes \& les Termes de toutes les Sciences et des Arts. La Haye-Rotterdam: Arnout \& Reinier, 1690.

FRIEDRICH, Carl Joachim. La démocratie constitutionnelle. Paris: Presses Universitaires de France, 1958.

GINZBURG, Carlo. Fear Reverence Terror. Reading Hobbes Today. Firenze: European University Institute, 2008.

GULLİ, Bruno. The Ontology and Politics of Exception: Reflections on the Work of Giorgio Agamben. (In) CALARCO Matthew; DeCAROLI, Steven (Org.). Giorgio Agamben. Sovereignty and Life. Standford, California: Standford University Press, 2007, p. 219-241.

HAMACHER, Werner. The Quaking of Presentation. (In: Id) Premises. Essays on Philosophy and Literature from Kant to Celan. Standford: Standford University Press, 1999, p. 261-293.

HARTOG, François. Anciens, Modernes, Sauvages. Paris: Galaade Éditions, 2005.

HUMPHREYS, Stephen. Legalizing Lawlessness: On Giorgio Agamben's State of Exception. The European Journal of International Law, v. 17, n. 3, p. 677-687, 2006.

JANSSEN, Laurens Franciscus. Abdicatio. Nieuve onderzoekingen over de dictatuur. Utrecht: Kemink \& Zoon,1960.

JASPERS, Karl. La bomba atómica y el futuro de la Humanidad. Traducción de Irene Garfeldt-Klever de Leal. Buenos Aires: Compañía General Fabril Editora, 1961.

KANT, Immanuel. Geschichte und Naturbeschreibung der merkwürdigsten Vorfälle des Erdbebens welches an dem Ende des 1755sten Jahres einen großen Theil der Erde erschüttert hat. (In) Kants Gesammelte Schriften. Edición de la Preussischen Akademie Wissenschaften, Berlin: de Gruyter, 1902, v. 1, p. 429-462. 
KIESOW, Rainer Maria. Ius sacrum. Giorgio Agamben und das nakte Recht. Rechtsgeschichte I, Heidelberg, 2002, p. 56-70.

KLEIST, Heinrich von. Das Erdbeben in Chili. (In: Id.) Werke und Briefe in vier Bänden. Edición de Siegfried Streller. Berlin-Weimar: Aufbau, 1978, p. 158-175.

LATOUR, Bruno. Face à Gaïa. Huit conférences sur le nouveau régime climatique. Paris: La Découverte, 2015.

LEAKEY, Richard; LEWIN, Roger. The Sixth Extinction. Patterns of Life and the Future of Humankind. New York: Anchor Books, 1995.

LEIBNIZ, Gottfried. Ensayos de Teodicea. Traducción de Miguel García Baró y Merecedes Huarte. Salamanca: Sígueme, 2013.

LEVI-STRAUSS, Claude. Tristes Trópicos. Traducción de Noelia Bastard. Barcelona: Paidós, 1988.

LIETZMANN, Hans. "Von der konstitutionellen zur totalitären Diktatur. Carl Joachim Friedrichs Totalitarismustheorie". In: SÖLLNER, Alfons; WALKENHAUS, Ralf; WIELAND, Karin (ed.). Totalitarismus. Eine Ideengeschichte des 20. Jahrhunderts. Berlin: Akademie Verlag, 1997, p. 174-194.

LITTRÉ, Émile. Dictionnaire de la langue française. París: Hachette, 1872-1877. MAQUIAVELO, Nicolás. Discursos sobre la primera década de Tito Livio. Traducción de Roberto Raschella. Buenos Aires: Losada, 2004.

MENANDRO. Heros. Theophoroumene. Karchedonios. Kitharistes. Kolax. Koneiazomenai. Leukadia. Misoumenos. Perikeiromene. Perinthia. Cambridge, MA: Harvard University Press, 1997.

NANCY, Jean-Luc. L'Équivalence des catastrophes (Après Fukushima). Paris: Éditions Galilée, 2012.

NISSEN, Adof. Das lustitium. Eine Studie aus der römischen Rechtsgeschichte. Leipzig: Gebhardt, 1877.

ORRÙ, Marco. Anomie. History and Meanings. London: Allen \& Unwin, 1987.

PARKER, Robert. Miasma. Pollution and Purification in Early Greek Religion. Oxford: Clarendon Press, 1983.

PLATÓN. Les Lois. Texto y traducción de Edouard des Places. Paris: Les Belles Lettres, 2006.

RECHENAUER, Georg. Thukydides und die hippokratische Medizin.

Naturwissenschaftliche Methodik als Modell für Geschichtsdeutung. Hildesheim; Zürich; New York: Georg Olms Verlag, 1991. 
REINACH, Théodore. De l'État de Siège. Étude historique et juridique. Paris: Librairie Cotillon, 1885.

RINGER, Fritz. The Decline of the German Mandarins. The German Academic Community, 1890-1933. Massachusetts: Harvard University Press, 1969.

ROSSITER, Clinton. Constitutional Dictatorship. Crisis Government in the Modern Democracies. Princeton: Princeton University Press, 1948.

SAINT-BONNET, François. L’État d'exception. París: Presses Universitaires de France, 2001.

SALERNO, Francesco. Dalla "conscratio" alla "publicatio bonorum". Forme giuridiche e uso politico dalle origini a Cesare. Napole: Jovene Editore, 1990.

SCHMITT, Carl. Die Diktatur. Von den Anfängen des modernen

Souveränitätsgedankens bis zum proletarischen Klassenkampf. Berlin: Duncker \& Humblot, 1994.

. Politische Theologie. Vier Kapitel zur Lehre von der Souveränität. Berlin: Duncker \& Humblot, 2015.

SCHRADER, Hans-Jürgen. Spuren Gottes in den Trümmern der Welt. Zur Bedeutung biblischer Bilder in Kleists 'Erdbeben'. Kleist-Jahrbuch, volumen 1, p. 27-42, 1991.

SCHÜTTE, Uwe. Köpfe knacken - 'Der Findling \& Das Erdbeben in Chili'. (In: Id) Die Poetik des Extremen. Ausschreitungen einer Sprache des Radikalen, Göttingen: Vandenhoeck \& Ruprecht, 2006, p. 97-110.

SEBALD, Georg, Winfried. Luftkrieg und Literatur. Mit einem Essay zu Alfred Andersch. Frankfurt am Main: Fischer Taschenbuch Verlag, 2001.

SLOTERDIJK, Peter. EI Reino de la Fortuna / Das Reich der Fortuna. Edición alemana y traducción castellana de Isidoro Reguera. Badajoz: Fundación Ortega Muñoz, 2013.

SPENGLER, Oswald. Der Untergang des Abendlandes. Umrisse einer

Morphologie der Weltgeschichte, Vollständige Ausgabe in einem Band. München: Beck, 1963.

STRINGER, Chris; DAVIS, William. Archaeology: those elusive Neanderthals. Nature, n. 413, p. 791-792, 2001.

TINGSTÉN, Herbert. Les Pleins Pouvoirs. L'expansion des pouvoirs gouvernamentaux pendant et après la grande guerre. Paris: Librairie Stock, 1934.

TUCÍDIDES. Historia de la Guerra del Peloponeso. Traducción de Francisco Romero Curz, Madrid: Cátedra, 2005. 
VOLTAIRE, François Marie Arouet de. Poème sur le désastre de Lisbonne. (In: Id.) Poésies, Paris: Les Belles Lettres, 2003.

WALKER, Rob. L'international, l'impérial, l'exceptionnel. Cultures \& Conflits.

Sociologie Politique de I'International, n. 58, p.13-51, 2005.

Artigo recebido em: 29/08/2017

Artigo aprovado em: 15/11/2017

Artigo publicado em: 18/12/2017 